Editorial

\title{
The Greening of IT: Paradox or promise?
}

It is said that we live in the anthropocene age (Crutzen and Stoermer, 2000) - from the Greek anthropo "human" and -cene- "new" - or the age when humans shape the earth. Thus the earth gave rise to humans and now humans are shaping the earth. However, this misses a critical aspect - technology. Just as the earth gave rise to us humans, we humans gave rise to our offspring: technology. And in time, just as the earth has shaped us, technology has become the means by which we shape the earth - and critically ourselves. Perhaps, as a consequence, it is more accurate to say that we live in the technopocene age (see Berthon, 2010).

Amongst our teaming technologies, that of information systems is perhaps preeminent, both directly (for example, the Internet alone accounts for $10 \%$ of energy consumption in the US) and indirectly (for example information systems enable most of the pollution producing industries world-wide) in terms of its facilitation of humankind's impact on both the ecosystem and sociosystem. It is perhaps trite to say that we live is a time of crisis, the media is awash with claims and counterclaims of our planet's ecological problems, yet it is an appropriate word. For the word crisis comes from the Greek krísis, and means not a time of peril (although arguably that is what we are in), but a time of decision. It is derived from the word krînein, meaning to decide, separate, judge. Thus, now is a time to decide on how we should employ the very technologies that have enabled and amplified not only the positive, strategic gains, but also, humankind's negative impact on the planet: the unintended consequences about which the likes of Robey and Boudreau (1999) speak in terms of IT's organizational impacts.

This is the focus of this special edition of JSIS. In some ways, it speaks to the kind of challenges set for us in Wastell and White's (2010) provocative Viewpoint article in last issue of JSIS; but it goes further than merely focusing on civic engagement. Green IT is a sobriquet that has been attached to a broad range of initiatives and technologies that have sought directly (in their embodiment) and indirectly (through facilitation) to monitor, control, and change our impact on the environment.

The study of Green IT, from a management decision-making perspective, can be thought of as comprising a number of dimensions or domains. In this special issue we focus on four key domains: regulations, technologies, assessment and consequences. First, from a socioeconomic and legal perspective, firms increasingly have to comply with a series of national and international environmental protocols; these range from government laws, through institutional standards, to voluntary codes of practice. Second, from a technological perspective, a myriad of new developments - at both component- and system-level - battle for managers' attention. Third, managers are faced with the challenge of assessment: which environmental protocols and which technologies should a firm adopt? Finally, there is the challenge of measuring the consequences of any adoption. These dimensions are summarized in Fig. 1.

Having outlined our framework, we are now in a position to introduce the eight papers that comprise this special issue. Addressing the regulatory dimension, Tom Butler's paper, "Compliance with Institutional Imperatives on Environmental Sustainability: Building Theory on the Role of Green IS", utilizes institutional and organizational theory to build and refine, using case data, a conceptual framework on how managers' enact compliance protocols.

In the technological domain, Leyland Pitt, Michael Parent, Iris Junglas, Anthony Chan and Stavroula Spyropoulou in, "Integrating the Smartphone into a Sound Environmental Information Systems Strategy: Principles, Practices and a Research Agenda", look at the rise of the smartphone (and concomitant applications or "apps") and its potential to enhance sustainable business strategies. The authors then provide a decision-making process to guide managers in identifying and selecting smartphone apps for sustainable issues.

Addressing the assessment domain we have four papers. First, Ranjit Bose and Xin Luo, in "Integrative Framework for Assessing Firms' Potential to Undertake - Green IT Initiatives via Virtualization - A Theoretical Perspective”, focus on how virtualization can be employed to assess an organization's ability to undertake green initiatives. They develop a model to help managers navigate such a process. The second of these papers moves from virtualization to reality. Rick Watson, Marie-Claude Boudreau, Adela Chen and Héctor Sepulveda, in "Green projects: An Information Drives Analysis of Four Cases”, assess a quartet of green transportation systems using their 'information drives' framework, which they extend to the physical domain. The model they develop can help managers assess the efficacy of a wide variety of green systems. 


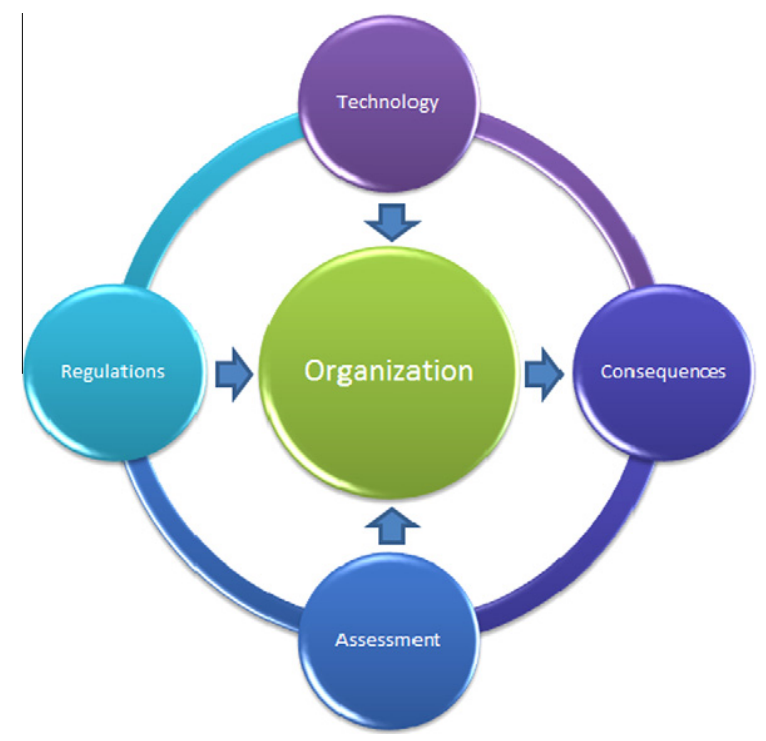

Fig. 1. Key dimensions of Green IS decision-making.

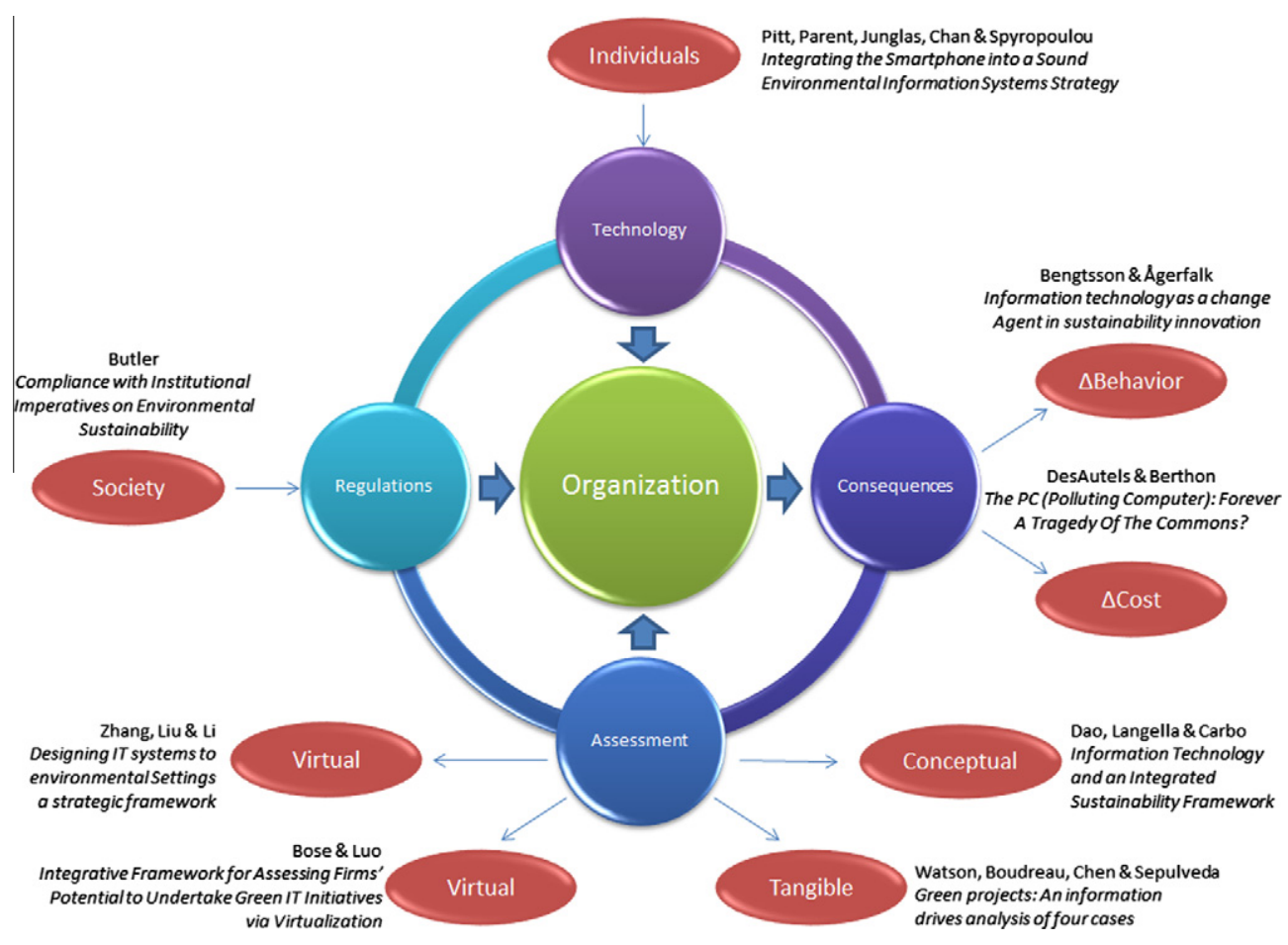

Fig. 2. Dimensions of Green IS decision-making and the papers.

In the third of the 'assessment' papers, "From Green to Sustainability: Information Technology and an Integrated Sustainability Framework", Dao, Longello and Carbo use the resource-based view of the firm to develop an integrated sustainability framework, illustrating the integration of human, supply chain, and IT resources to enable firms develop sustainability capabilities. The fourth and final paper on this theme, from Zhang, Liu and Li, "Designing IT systems according to environmental settings: a strategic analysis framework" employs the goal-oriented requirements language, GRL, to model the rationality behind IT system design, and in particular, considers how environment-related considerations come into play in design decision making. 
As regards the consequences dimension, we have our final two papers. In the first paper, Fredrik Bengtsson and Pär J. Ågerfalk, in "Information technology as a change actant in sustainability innovation: Insights from Uppsala", look at the changes that occur due to the implementation of an information system designed specifically to enhance sustainability in the Swedish municipality of Uppsala. Acton Network Theory is employed to show how information systems can become powerful agents of change. The second of these papers, by Philip DesAutels and Pierre Berthon, "The PC (Polluting Computer): Forever a Tragedy of The Commons?" looks at the monetary consequences of complying with sustainability standards. Using the tragedy of the commons framework, they hypothesise that the cost of adopting sustainability standards will result in higher prices; intriguingly their study finds otherwise and explores reasons why.

The eight papers are summarized in Fig. 2.

As these papers suggest - the road ahead for Green IT is full of promise - and perils. Despite the best intentions, many information systems projects have magnified the very problems that they were designed to solve: consider the paperless office (which resulted in more paper), for instance, or the one lap-top per child (which has resulted in a deterioration in child learning), or the HIT (industry shorthand for 'health-information technology') project (which early tests have indicated a resultant deterioration in healthcare outcomes, cf., Berthon et al., 2011). This is not to sound dystopian - but IS managers must realize that any system produces pleiotropic effects: we need to be watchful of the systems we create and how they change us and the planet. The technopocene requires a new level of mindfulness on the part of humans for themselves and their technological offspring.

\section{References}

Berthon, P., 2010. The 'Technopocene' - The Rise of Technology, Working Paper, Bentley University, Waltham, MA, USA.

Berthon, P., Desautels, P., Donnellan, B., Clark-Williams, C., 2011. Green digits: towards an ecology of IT thinking. In: Galliers, R.D., Currie, W.L. (Eds.), The Oxford Handbook of Management Information Systems: Critical Perspectives and New Directions. Oxford University Press, Oxford, UK.

Crutzen, P.J., Stoermer, E.F., 2000. The 'Anthropocene'. Global Change Newsletter 41, 17-18.

Robey, D., Boudreau, M.C., 1999. Accounting for the contradictory organizational consequences of information technology: theoretical directions and methodological implications. Information Systems Research 10 (2), 167-185.

Wastell, D., White, S., 2010. Facts, myths and thought-styles. . . and a rallying cry for civic engagement. Journal of Strategic Information Systems 19 (4), 307318.

Pierre Berthon Bentley University, USA E-mail address: pberthon@bentley.edu

Brian Donnellan National University of Ireland, Maynooth, Ireland E-mail address: brian.donnellan@nuim.ie

Available online 23 February 2011 